\title{
You cannot assess until you measure it: using psoas index for measuring frailty
}

\author{
Ho Jin Kim
}

Department of Thoracic and Cardiovascular Surgery, Asan Medical Center, University of Ulsan College of Medicine, Seoul, Korea

Correspondence to: Ho Jin Kim, MD. Department of Thoracic and Cardiovascular Surgery, Asan Medical Center, University of Ulsan College of Medicine, 88, Olympic-ro 43-gil, Songpa-gu, Seoul 05505, Korea. Email: hjkim1978@amc.seoul.kr.

Provenance: This is an invited Editorial commissioned by the Executive Editor-in-Chief Jianxing He (Department of Cardiothoracic Surgery, The First Affiliated Hospital of Guangzhou Medical University, Guangzhou, China).

Comment on: Hawkins RB, Mehaffey JH, Charles EJ, et al. Psoas Muscle Size Predicts Risk-Adjusted Outcomes After Surgical Aortic Valve Replacement. Ann Thorac Surg 2018;106:39-45.

Submitted Nov 09, 2018. Accepted for publication Nov 22, 2018.

doi: $10.21037 /$ jtd.2018.11.113

View this article at: http://dx.doi.org/10.21037/jtd.2018.11.113

Due to the increasing incidence of more complex heart diseases with the aging population, cardiac surgeons are having difficulties in determining who is most likely to benefit from a surgical intervention with a minimum risk of mortality and morbidity. In particular, the assessment of operative risk has become more critical in patients with severe aortic stenosis, owing to the successful employment of alternative transcatheter therapy. Several risk prediction models, such as the Society of Thoracic Surgeons (STS) Predicted Risk of Mortality and Morbidity and the European System for Cardiac Operative Risk Evaluation (euroSCORE) II, have been widely used to provide relevant information to cardiac surgeons for the assessment of the risk of cardiac surgery.

Although these models have been known to be useful for predicting the perioperative risk of mortality and morbidity after cardiac surgery, they have been criticised for not adequately reflecting frailty in patients in their risk stratification algorithms (1). Frailty can be defined as decreased physiological reserve across the multiple physiologic systems to adapt to the stressors, such as major cardiac surgery (2). Frail patients may be at higher risk of postoperative mortality than those not deemed frail despite seemingly similar comorbidities. As assessing the likelihood of surgical benefits in the high-risk cohort is increasingly important, frailty has been considered by cardiac surgeons as a significant factor during the preoperative risk evaluation (1).
Although the importance of frailty is increasingly recognised during the risk assessment for cardiac surgery, its incorporation into the risk models has been limited due to the lack of agreed measurement methods. This may be attributed to the absence of consensus definition of frailty that led cardiac caregivers to measure some of its limited aspects based on clinical or research interests. In a neighbouring study conducted on the high-risk cohorts undergoing surgical or transcatheter aortic valve replacement (SAVR or TAVR, respectively), frailty was measured as a composite of grip strength, gate speed, serum albumin, and activities of daily living (3). In another TAVR study with the use of self-expandable prostheses, anaemia or body mass index was also evaluated as a frailty parameter (4). Such attempts to comprehensively assess frailty may be time- and labour-intensive, which limits the availability of frailty measures in a busy real-world practice. In this regard, objective, reproducible, and easy to assess frailty measures are needed.

In light of these issues, Hawkins and colleagues suggested the use of the psoas muscle cross-sectional area to measure frailty based on the hypothesis that sarcopenia manifested by decreased psoas muscle area is predictive of increased mortality, morbidity, and resource utilisation (5). The cross-sectional area of the psoas muscle was obtained by measuring at the L4 vertebral level from preoperative computed tomography (CT) scans in 240 moderateto-high-risk patients (median age, 80 years; 40 women) 
undergoing SAVR whose STS predictive risk of mortality is $>3 \%$. The cross-sectional area of the psoas muscle was further refined to be indexed based on the body surface area (psoas index). Patients were identified to have sarcopenia when their psoas index was below the 25 th sex-specific percentile. The authors demonstrated that the psoas index is predictive of 1-year mortality, STS major morbidity, and increased resource utilisation such as hospital cost or length of hospital stay.

The patients in this study were selected based on the availability of preoperative CT scan. Consequently, among the total of 649 high-risk patients, only 240 were enrolled, and the selection bias may be a significant issue accordingly. Besides, reduced sample size of the study cohort may have significantly limited the statistical power to show the differences in early and long-term clinical outcomes between the sarcopenic and nonsarcopenic groups; the only statistically significant difference between the two groups was 1 -year mortality $(\mathrm{P}=0.029)$. Because there is no widely accepted definition of sarcopenia based on psoas index, the authors defined sarcopenia as a psoas index of $<25$ th percentile in the study cohort, not referenced by the normal range obtained from the general population. In this regard, under- or misdiagnosis of patients with sarcopenia is possible. The main results based on grouping the patients into 'sarcopenic' and 'nonsarcopenic' may have been changed according to the introduction of new psoas index criteria to define sarcopenia.

Nonetheless, the authors are to be commended for initiating a study that suggests the clinical significance of psoas index as an indicator of frailty. They showed that increment of the psoas index was associated with reduced risk of 1-year mortality and hospital resource use. Since psoas index can be objectively and reproducibly measured, its use as an indicator of frailty is to be further validated in future studies with larger cohorts. As agreed by the authors, whether psoas index can comprehensively measure frailty remains controversial. The use of psoas index would be better addressed with the precise definition of sarcopenia based on psoas index criteria, encompassing a wide range of heart surgeries not confined to SAVR. As more studies on the association between the psoas index and the risk of cardiac surgery are available, the use of psoas index as a predictor of frailty will have more solid grounds.

\section{Acknowledgements}

None.

\section{Footnote}

Conflicts of Interest: The author has no conflicts of interest to declare.

\section{References}

1. Paknikar R, Friedman J, Cron D, et al. Psoas muscle size as a frailty measure for open and transcatheter aortic valve replacement. J Thorac Cardiovasc Surg 2016;151:745-51.

2. Partridge JS, Harari D, Dhesi JK. Frailty in the older surgical patient: a review. Age Ageing 2012;41:142-7.

3. Green P, Arnold SV, Cohen DJ, et al. Relation of frailty to outcomes after transcatheter aortic valve replacement (from the PARTNER trial). Am J Cardiol 2015;116:264-9.

4. Adams DH, Popma JJ, Reardon MJ, et al. Transcatheter aortic-valve replacement with a self-expanding prosthesis. N Engl J Med 2014;370:1790-8.

5. Hawkins RB, Mehaffey JH, Charles EJ, et al. Psoas Muscle Size Predicts Risk-Adjusted Outcomes After Surgical Aortic Valve Replacement. Ann Thorac Surg 2018;106:39-45.
Cite this article as: Kim HJ. You cannot assess until you measure it: using psoas index for measuring frailty. $\mathrm{J}$ Thorac Dis 2018;10(12):6412-6413. doi: 10.21037/jtd.2018.11.113 